\title{
Incompletely resected non-small cell lung cancer: Do we know how to handle a positive margin?
}

\author{
Bryan M. Burt, MD
}

\footnotetext{
From the Division of General Thoracic Surgery, Department of Surgery, Baylor College of Medicine, Houston, Tex

Disclosures: Author has nothing to disclose with regard to commercial support.

Received for publication April 2, 2017; accepted for publication April 4, 2017; available ahead of print May 16 2017.

Address for reprints: Bryan M. Burt, MD, Division of General Thoracic Surgery, Department of Surgery, Baylor College of Medicine, One Baylor Plaza, BCM 390, Houston, TX 77005 (E-mail: bryan.burt@bcm.edu).

J Thorac Cardiovasc Surg 2017;154:673-4

$0022-5223 / \$ 36.00$

Copyright (C) 2017 Published by Elsevier Inc. on behalf of The American Association for Thoracic Surgery http://dx.doi.org/10.1016/j.jtcvs.2017.04.009
}

The National Comprehensive Cancer Network (NCCN) clinical practice guidelines ${ }^{1}$ are a statement of evidence and consensus of an expert panel of authors regarding their views of currently accepted approaches to treatment. The NCCN clinical practice guidelines document is an authoritative pillar in what constitutes the "standard of care" in medicine. In cancer research, the quality of evidence that exists is highly variable, and the highest level of evidence (randomized, controlled trials) is not available to support recommendations in most clinical situations. Much of the evidence that shapes best clinical practice is therefore based on data from indirect comparisons among nonrandomized clinical trials, retrospective studies, and clinical observations. Furthermore, there are many common clinical scenarios in which we have no meaningful clinical data to support clinical management, and for which clinical experience is the bedrock platform for such decision making. In these cases, the NCCN provides recommendations that are based on both the level of the available clinical evidence and the degree of consensus among a designated panel of cancer specialists and clinical experts.

There are surprisingly few data on the management of patients with positive margins after resection for non-small cell lung cancer (NSCLC). This is particularly true for early-stage disease (stage IA), for which the NCCN recommendations of postoperative radiotherapy (PORT) are based on low-level evidence and for which there is a not a uniform NCCN panel consensus that the intervention is appropriate (a category $2 \mathrm{~B}$ recommendation). ${ }^{1}$ In this issue of the Journal, Smeltzer and colleagues ${ }^{2}$ have addressed our knowledge gap in this area through thoughtful analyses of the National Cancer Database, a robust data set sourced from Commission on Cancer-accredited facilities that covers approximately $70 \%$ of newly diagnosed cancer cases in the United States. The objective of Smeltzer and colleagues $^{2}$ was validation of the NCCN adjuvant therapy guidelines after incomplete resection of NSCLC in 3461 patients with R1 or R2 resections, and their findings were striking. The current NCCN recommendation for patients

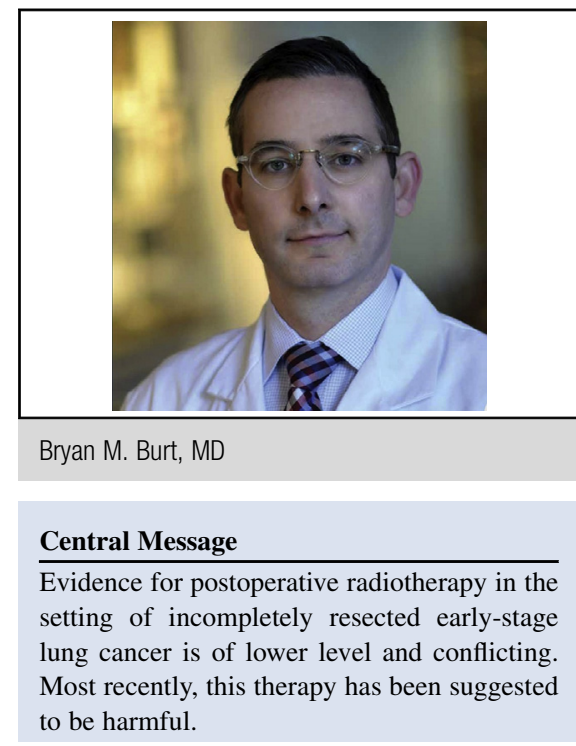

See Article page 661

with incompletely resected stage IA (T1a, N0) NSCLC who cannot undergo repeated resection is PORT; however, Smeltzer and colleagues ${ }^{2}$ demonstrated that this cohort had a significantly worse outcome with PORT than without treatment (5-year overall survivals of $26 \%$ vs $58 \%$, respectively). Similarly, for patients with incompletely resected stage IB/IIA (N0) NSCLC, the current NCCN recommendation for patients who cannot undergo repeated resection is PORT with or without chemotherapy. Evaluation of this cohort in the National Cancer Database demonstrated again that radiotherapy was associated with significantly decreased 5-year overall survival relative to those patients left untreated ( $25 \%$ vs $47 \%$ ).

The first point that must be emphasized to our readers is that repeated resection is the preferred treatment for incompletely resected early stage NSCLC (stage IA-IIA). Smeltzer and colleagues ${ }^{2}$ excluded patients who underwent repeated resections from their study cohort to facilitate the investigation of other postoperative modalities. Next, the data of Smeltzer and colleagues ${ }^{2}$ suggest that PORT was harmful to patients with incompletely resected early-stage NSCLC, and these observations do not support-and in fact contest-our current NCCN guidelines. This observed lack of benefit of PORT in patients with incompletely resected early-stage NSCLC is similar to a body of current evidence (also of lower level) in patients with completely resected early-stage NSCLC. ${ }^{3,4}$ It thus may be true that 
any positive influence of PORT on overall survival does not overcome its treatment-associated mortality risk. These data are compelling and derived from a well-executed study; however, they are limited, retrospective, and still considered as lower level evidence. To highlight one limitation, the details regarding the mode of radiotherapy are not provided, and it is somewhat surprising to see an actual survival detriment in the PORT group in the era of stereotactic ablative radiotherapy, which is very well tolerated. As is the case for most clinical scenarios, it is unlikely that data from a randomized, controlled trial will ever be available to support the answer to this question, given the relative infrequency of incomplete resection ( $4 \%$ in this study). We therefore will continue to rely on well-designed comparative studies such as this one and on expert opinion to formulate our recommendations, and this study will likely be considered in formulation of the next iteration of the NCCN guidelines.

\section{References}

1. National Comprehensive Cancer Network. The NCCN Clinical Practice Guidelines in Oncology (NCCN Guidelines). Non-small cell lung cancer (version 4.2017). Available at: http://www.nccn.org/professionals/physician_gls/f_guidelines.asp\#nscl. Accessed March 1, 2017.

2. Smeltzer MP, Lin CC, Kong FM, Jemal A, Osarogiabon RU. Survival impact of post-operative therapy modalities according to margin status in non-small cell lung cancer patients in the United States. J Thorac Cardiovasc Surg. 2017;154: 661-72.

3. Postoperative radiotherapy in non-small-cell lung cancer: systematic review and meta-analysis of individual patient data from nine randomised controlled trials. PORT Meta-analysis Trialists Group. Lancet. 1998;352: 257-63.

4. Burdett S, Stewart L, PORT Meta-analysis Group. Postoperative radiotherapy in non-small-cell lung cancer: update of an individual patient data meta-analysis. Lung Cancer. 2005;47:81-3. 